\title{
Towards more accurate and reliable predictions for nuclear applications
}

\author{
Stephane Goriely ${ }^{1, \text { a }}$, Stephane Hilaire ${ }^{2}$, Noel Dubray ${ }^{2}$, and Jean-François Lemaître ${ }^{1}$ \\ 1 Institut d'Astronomie et d'Astrophysique, Université Libre de Bruxelles, CP-226, 1050 Brussels, Belgium \\ 2 CEA, DAM, DIF, 91297 Arpajon, France
}

\begin{abstract}
The need for nuclear data far from the valley of stability, for applications such as nuclear astrophysics or future nuclear facilities, challenges the robustness as well as the predictive power of present nuclear models. Most of the nuclear data evaluation and prediction are still performed on the basis of phenomenological nuclear models. For the last decades, important progress has been achieved in fundamental nuclear physics, making it now feasible to use more reliable, but also more complex microscopic or semimicroscopic models in the evaluation and prediction of nuclear data for practical applications. Nowadays mean-field models can be tuned at the same level of accuracy as the phenomenological models, renormalized on experimental data if needed, and therefore can replace the phenomenological inputs in the evaluation of nuclear data. The latest achievements to determine nuclear masses within the non-relativistic HFB approach, including the related uncertainties in the model predictions, are discussed. Similarly, recent efforts to determine fission observables within the mean-field approach are described and compared with more traditional existing models.
\end{abstract}

\section{Introduction}

Among the various fields in nuclear astrophysics, nucleosynthesis is clearly the one the most closely related to nuclear physics, the nuclear physics imprint being found in the origin of almost all nuclides produced in the Universe [1]. Impressive progress has been made for the last decades in the various fields related to nucleosynthesis, especially in experimental and theoretical nuclear physics, as well as in ground-based or space astronomical observations and astrophysical modellings. In spite of that success, major problems and puzzles remain. In particular, experimental nuclear data only covers a minute fraction of the whole set of data required for nucleosynthesis applications. Reactions of interest often concern unstable or even exotic (neutronrich, neutron-deficient, superheavy) species for which no experimental data exist. In addition, a large number (thousands) of unstable nuclei may be involved for which many different properties have to be determined (Fig. 1). Finally, the energy range for which measurements are available is restricted to the small range reachable by present experimental setups. To fill the gaps, only theoretical predictions can be used.

Among the various nuclear astrophysics problems, one specific nucleosynthesis process remains extremely difficult to solve. It concerns the rapid neutron-capture process, or r-process, invoked to explain the production of the stable (and some long-lived radioactive) neutronrich nuclides heavier than iron that are observed in stars of various metallicities, as well as in the solar system (for a review, see Ref. [2]). In recent years,

a e-mail: sgoriely@astro.ulb.ac.be nuclear astrophysicists have developed more and more sophisticated r-process models, trying to explain the solar system composition in a satisfactory way by adding new astrophysical or nuclear physics ingredients. The r-process remains the most complex nucleosynthetic process to model from the astrophysics as well as nuclearphysics points of view. The site(s) of the r-process is (are) not identified yet, all the proposed scenarios facing serious problems. Complex — and often exotic — sites have been considered in the hope of identifying astrophysical conditions in which the production of neutrons is large enough to give rise to a successful r-process.

Progress in the modelling of type-II supernovae and $\gamma$-ray bursts has raised a lot of excitement about the so-called neutrino-driven wind environment [2-4]. However, until now a successful r-process has not been obtained $a b$ initio without tuning the relevant parameters (neutron excess, entropy, expansion timescale) in a way that is not supported by the most sophisticated existing models. Early in the development of the theory of nucleosynthesis, an alternative to the r-process in hightemperature supernova environments was proposed. It concerns the decompression of cold neutron star (NS) matter which was found to be favorable for strong r-processing [5].

Recently, special attention has been paid to NS mergers following the confirmation by hydrodynamic simulations that a non-negligible amount of matter, typically about $10^{-3}$ to $10^{-2} \mathrm{M}_{\odot}$, can be ejected. In contrast to the supernova site, investigations with growing sophistication have confirmed NS merger ejecta as viable sites for strong r-processing [6, and references therein]. In particular, recent nucleosynthesis calculations [6] show that the combined contribution of both the dynamical (prompt) 


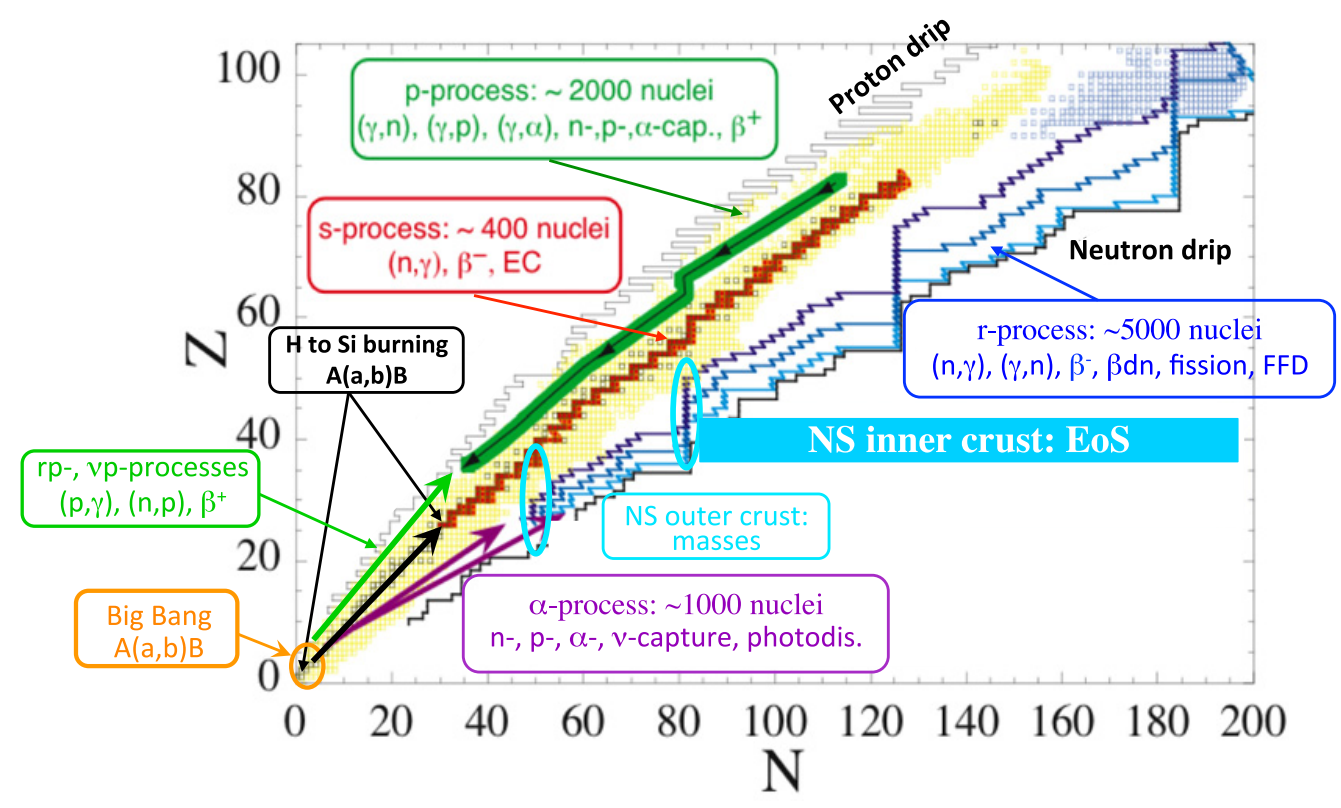

Figure 1. Schematic representation in the $(N, Z)$ plane of the different low-energy nuclear astrophysics applications, including nucleosynthesis processes, composition and structure properties of neutron stars. For each process, the nuclear needs are sketched. The open black squares correspond to stable or long-lived nuclei, the yellow squares to the nuclei for which masses have been measured and the blue square to those which may fission.

ejecta expelled during the binary NS-NS or NS-black hole $(\mathrm{BH})$ merger, as well as the neutrino and viscously driven outflows generated during the post-merger remnant evolution of the relic BH-torus systems lead to the production of r-process elements from $A \gtrsim 90$ up to thorium and uranium with an abundance distribution that reproduce extremely well the solar distribution, as well as the elemental distribution observed in low-metallicity stars [8]. Note, however, that the relativistic NS-NS merger simulations of Ref. [9] for a soft nuclear equation of state shows a significant impact of neutrino reactions on the electron fraction in the dynamical ejecta of cases with delayed collapse of the merger remnant. In this case, in addition to heavy r-process elements $(A>140)$, nuclei with lower mass numbers may also be created in the dynamical ejecta. It is still unclear whether the observed effects apply similarly strongly to all high-density equation of states and all binary systems that lead to a delayed collapse of the merger remnant.

The ejected mass of r-process material, combined with the predicted astrophysical event rate (around $10 \mathrm{Myr}^{-1}$ in the Milky Way [10]) can account for the majority of r-material in our Galaxy [7]. Recent studies [11, and references therein] have also reconsidered the galactic or cosmic chemical evolution of $r$ process elements in different evolutionary contexts, and although they do not converge towards one unique quantitative picture, most of them got to the conclusion that double compact star mergers may be the major production sites of r-process elements.

In this specific r-process scenario, the number of free neutrons per seed nuclei can reach a few hundreds. With such a neutron richness, heavy fissioning nuclei can be produced. Thanks to this property, the final composition of the ejecta is rather insensitive to details of the initial abundances and the astrophysical conditions, in particular the mass ratio of the two NSs, the quantity of matter ejected, and the equation of state [7]. This robustness, which is compatible with the uniform, solarlike abundance pattern of the rare-earth elements observed in metal-poor stars [8], supports the possible creation of these elements by fission recycling in NS merger ejecta.

R-process nucleosynthesis calculations require a reaction network including about 5000 species from protons up to $\mathrm{Z} \gtrsim 110$ lying between the valley of $\beta$-stability and the neutron drip line. All chargedparticle fusion reactions on light and medium-mass elements that play a role when the nuclear statistical equilibrium freezes out need to be included in addition to radiative neutron captures and photodisintegrations. On top of these reactions, $\beta$-decays as well as $\beta$-delayed neutron emission probabilities and $\alpha$-decay rates need to be taken into account, but also fission processes, including neutron-induced, spontaneous, $\beta$-delayed and photofission, together with the corresponding fission fragment distribution for all fissioning nuclei. All rates are based on experimental information whenever available, but since only a extremely small amount of data are known experimentally, theoretical models are fundamental in providing the various predictions.

For such applications, the necessary ingredients (properties of cold and hot nuclei, nuclear level densities, optical potentials, $\gamma$-ray strength functions, fission properties, $\beta$-strength functions) should ideally be derived from global, universal and microscopic models. The large number of nuclides involved in the modelling of some nucleosynthesis mechanisms demands the use of global models. On the other hand, a universal description of all nuclear properties within a unique framework for all nuclei involved ensures the essential coherence of the predictions of all unknown data. Finally, a microscopic description provided by a physically sound theory based on first principles ensures extrapolations away from experimentally known energy or mass regions that are 
likely to be more reliable than predictions derived from more or less parametrized approaches of various types and levels of sophistication. Nowadays, microscopic models can be tuned to the same level of accuracy as the phenomenological models, and therefore could replace the phenomenological inputs in practical applications.

Today, due to our ignorance on the exact conditions in which the r-process takes place, it remains difficult to estimate the precision with which the various relevant rates need to be estimated. In particular, it strongly depends if an $(n, \gamma)-(\gamma, n)$ equilibrium would be reached during the neutron irradiation or if, instead, a competition between neutron captures and $\beta$-decays would be responsible for the nuclear flow and final shaping of the r-abundance distribution. Much more work on the astrophysical modelling [2] is needed before providing such constraints that could shed light on the precision required from nuclear physics. In the meantime, a first educated guess would require the reaction rates to be estimated within a factor of 2 and $\beta$-decay rates within $50 \%$ for all nuclei that may be direct progenitors of r-nuclei, i.e. before the final $\beta$-decay cascade at the neutron freeze-out. Concerning the more exotic nuclei up to the neutron drip-line, depending if fission efficiently recycles material, i.e. depending on the number of neutrons per seed available, less stringent constraints could be envisioned. It also remains of first importance to estimate the statistical as well as systematic uncertainties affecting the predictions far away from the experimentally known region. Such a difficult task has been started regarding mass predictions, but remains to be performed for the reaction as well as $\beta$-decay rates. Our capacity to predict two fundamental nuclear ingredients, namely nuclear masses and fission barriers, are discussed in the present paper in Sects. 2 and 3, respectively, and the need to improve global, universal and microscopic models emphasized.

\section{Nuclear masses}

Among the ground state properties, the atomic mass is obviously the most fundamental quantity. The calculation of the reaction cross section also requires the knowledge of other ground state properties, such as the deformation, density distribution or the single-particle level scheme. When not available experimentally, these quantities need to be extracted from a mass model which aims at reproducing measured masses as accurately as possible, i.e typically with a root-mean-square (rms) deviation of about $700 \mathrm{keV}$. The importance of estimating all ground state properties reliably should not be underestimated. For example, the nuclear level densities of a deformed nucleus at low energies (typically at the neutron separation energy) is predicted to be significantly (about 30 to 50 times) larger than those of a spherical one due principally to the rotational enhancement. An erroneous determination of the deformation can therefore lead to large errors in the estimate of radiative capture cross sections. For this reason, modern mass models not only try to reproduce at best experimental masses and mass differences, but also charge radii, quadrupole moments, giant resonances, fission barriers, shape isomers, infinite nuclear matter properties, ....

With a view to their astrophysical application in neutron-rich environments, a series of nuclear-mass

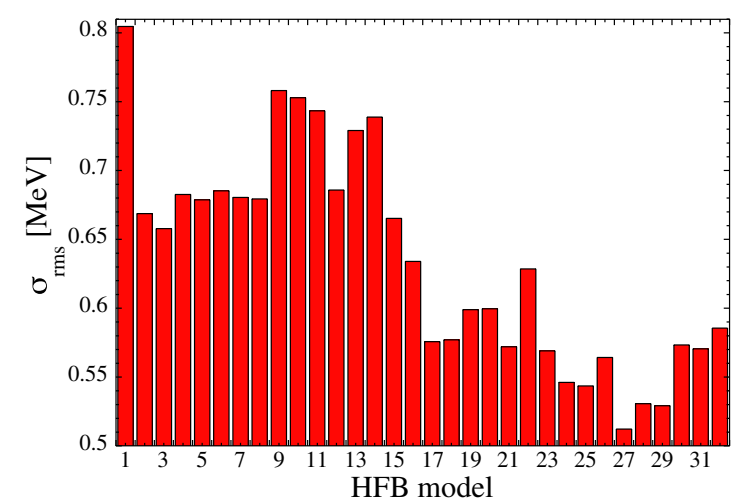

Figure 2. Representation of the rms deviation for the 32 SkyrmeHFB mass models, labeled from HFB-1 to HFB-32. The rms deviation is calculated with respect to the 2353 measured masses [13].

models have been developed recently based on the Hartree-Fock-Bogoliubov (HFB) method with Skyrme and contact-pairing forces, together with phenomenological Wigner terms and correction terms for the spurious collective energy within the cranking approximation [12, and references therein]; all the model parameters have been fitted to essentially all the experimental mass data. While the first HFB-1 mass model aimed at proving that is was possible to reach a low rms deviation with respect to all experimental masses available at that time, most of the subsequent models were developed to further explore the parameter space widely or to take into account additional constraints. These include in particular a sensitivity study of the mass model accuracy and extrapolation to major changes in the description of the pairing interaction, the spin-orbit coupling or the nuclear matter properties, such as the effective mass, the symmetry energy and the stability of the equation of state.

With respect to the 2353 measured masses [13], the 32 HFB mass models give an rms deviation ranging between $0.50 \mathrm{MeV}$ for HFB-27 and $0.79 \mathrm{MeV}$ for HFB-1, as illustrated in Fig. 2. These rms deviations can be compared to those obtained with other global mass model, such as the Gogny-HFB mass model with the D1M interaction [14] characterised by an rms of $0.79 \mathrm{MeV}$ or the 2012 version of the finite-range droplet model [15] with $0.58 \mathrm{MeV}$. However, when dealing with exotic nuclei far away from stability, deviations between the HFB mass predictions can become significant, not only in the rigidity of the mass parabola, but also in the description of the shell gaps or pairing correlations [16]. The $1 \sigma$ variance between the 32 HFB mass predictions (with respect to the HFB-24 mass model) are illustrated in Fig. 3 where deviations up to about $3 \mathrm{MeV}$ can be found at the neutron drip line for the heaviest species. Such uncertainties can be interpreted as the model uncertainties (due to model defects) inherent to the given HFB model [17] and are considered to be independent of parameter uncertainties. These model uncertainties have been shown to be significantly larger than the uncertainties associated with local variations of the model parameters in the vicinity of an HFB minimum [16], as estimated using a variant of the BackwardForward Monte Carlo method [18] to propagate the uncertainties on the masses of exotic nuclei far away from the experimentally known regions. 


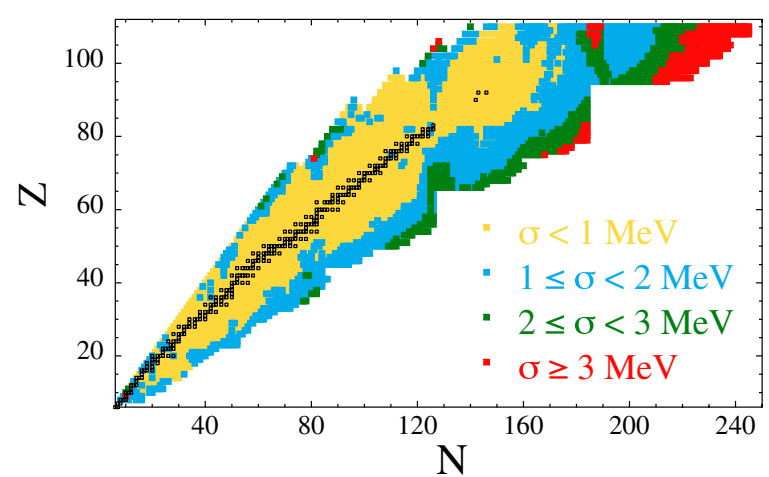

Figure 3. Representation in the $(N, Z)$ plane of the $1 \sigma$ uncertainty corresponding to the 32 Skyrme-HFB mass models for all the 8500 nuclei included in the mass tables from $Z=8$ up to $\mathrm{Z}=110$.

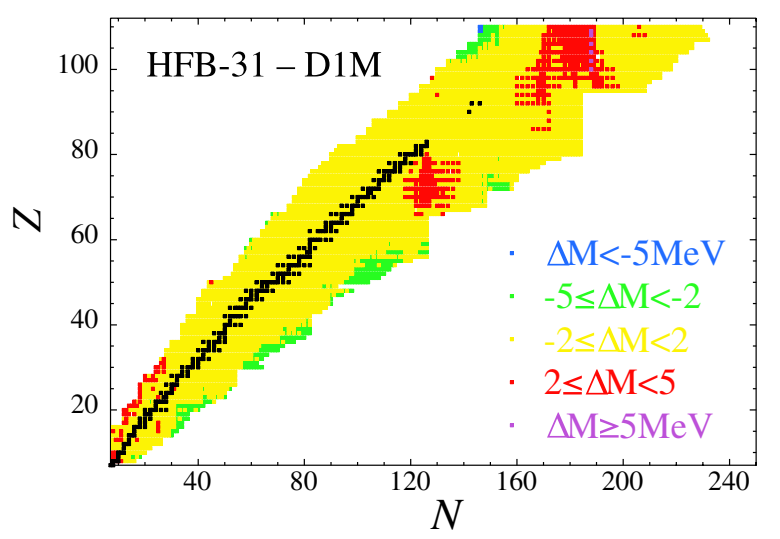

Figure 4. Representation in the $(N, Z)$ plane of the mass difference between HFB-31 [12] and the D1M [14] models for all the 8500 nuclei from $Z=8$ up to $Z=110$ between the HFB31 proton and neutron driplines.

Many effective interactions have been proposed to estimate nuclear structure properties within the relativistic or non-relativistic mean-field approach [19]. Except the BSk forces at the origin of the above-mentioned HFB mass models and the D1M interaction at the origin of the GognyHFB mass model [14], none of the others have been fitted to a large set of experimental masses. Consequently, their predictions lead to rms deviations typically larger than 2-3 MeV with respect to the bulk of known masses (e.g. masses obtained with the SLy4 force give an rms deviation of the order of $5 \mathrm{MeV}$ [20]). With such a low accuracy, these masses should not be used for applications, such as the r-process nucleosynthesis. Additionally, other global mass models have been developed, essentially within the macroscopic-microscopic approach [15,21] but remain unstable with respect to parameter variations, as shown in the framework of the droplet model in Ref. [22]. For this reason, more fundamental approaches are needed for practical applications.

When considering mass models obtained in relatively different frameworks, e.g the Skyrme-HFB or Gogny-HFB mass models, significantly larger deviations are found in the mass predictions away from the experimentally known region. For example, as shown in Fig. 4, deviations up to typically $\pm 5 \mathrm{MeV}$ are found for exotic neutronrich nuclei between HFB-31 [12] and D1M [14] mass predictions, especially around the $N=126$ and 184 shell closures. Neutron capture rates can consequently deviate by 3 to 5 orders of magnitude with such mass differences, essentially due to different local variations in the pairing and shell description. Such deviations by far exceeds what is acceptable for nucleosynthesis applications. For this reason, further improvements of the mass model are needed. These include development of relativistic as well as non-relativistic mean field models, but also the inclusion within such approaches of the state-of-the-art corrections, like the quadrupole or octupole correlations by the Generator Coordinate Method and a proper treatment of odd- $A$ and odd-odd nuclei with time-reversal symmetry breaking. Such models should reproduce not only nuclear masses at best, but also as many experimental observables as possible. These include charge radii and neutron skin thicknesses, fission barriers and shape isomers, spectroscopic data such as the $2^{+}$energies, moments of inertia, but also infinite (neutron and symmetric) nuclear matter properties obtained from realistic calculations as well as specific observed or empirical properties of neutron stars, like their maximum mass or mass-radius relations [23].

\section{Fission}

Almost all existing evaluations of the neutron-induced fission cross sections rely on the multiple-humped fission penetration model where barriers are described by inverted decoupled parabolas. Such approaches consider all ingredients as free parameters in order to be able to achieve more or less accurate fits to experimental cross sections. Although such adjustments respond to the needs of some nuclear applications, their predictive power remains poor due to the large number of free parameters. Such methods should not be used in applications requiring a purely theoretical description of fission for experimentally unknown nuclei, such as nuclear astrophysics. Recent studies aim at providing sounder descriptions of some of the basic nuclear ingredients required to describe fission cross sections. These concern in particular fission barriers (or more generally fission paths) and nuclear level densities at the fission saddle points, but also the fission fragment distribution, including the average number of emitted neutrons. Recently, such nuclear ingredients have been systematically determined in the framework of mean field models based on the Skyrme interaction, as reviewed in Refs. [24, 25].

New calculations of fission properties within the nonrelativistic HFB approach with the finite-range Gogny interaction are now also available [26-28]. They include detailed estimates of the potential energy surface with the D1M Gogny interaction [14] in the quadrupole-octupole deformation plane with triaxial corrections for the inner barrier. Quadrupole correlation energies are also taken into account. The HFB-D1M calculation is found to reproduce the 14 even-even empirical primary fission barriers [29] with an rms deviation of $0.49 \mathrm{MeV}$ to be compared with $0.75 \mathrm{MeV}$ for the HFB-14 model [24]. Accurate results with an rms of $0.35 \mathrm{MeV}$ are also obtained within the relativistic mean-field approach [30] which presents the advantage of breaking both the reflection and the axial symmetries simultaneously. In this framework, triaxial deformation is found to lower the outer barrier by 0.5 to $1 \mathrm{MeV}$. 


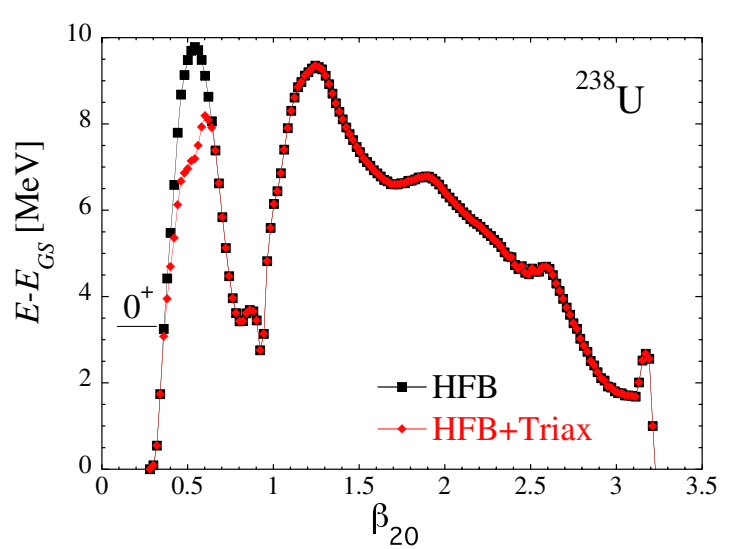

Figure 5. ${ }^{238} \mathrm{U}$ fission path as a function of the quadrupole deformation parameter $\beta_{20}$. The black squares correspond to the axially symmetric HFB-D1M path while the red diamonds includes the effect of triaxiality on the inner barrier. The $0^{+}$level shows the position of the ground-state zero-point energy.

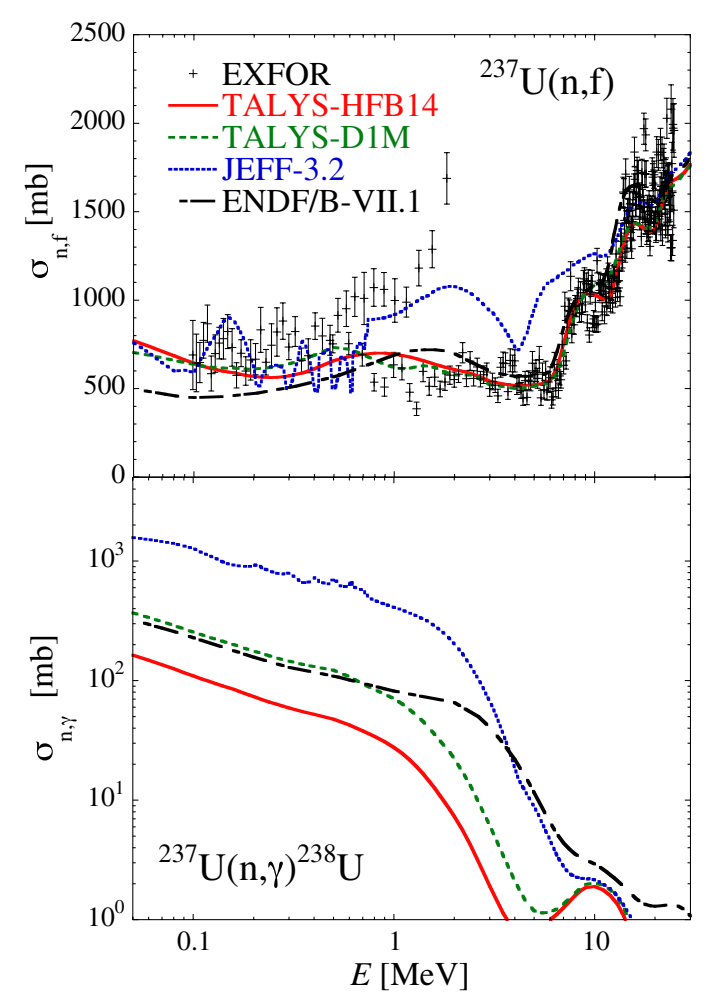

Figure 6. Upper panel: ${ }^{237} \mathrm{U}$ neutron-induced fission cross section obtained with the HFB-14 fission path (TALYS-HFB14) [31], the new D1M fission paths (TALYS-D1M), or the phenomenological fission properties in the JEFF-3.2 [32,33] or ENDF/B-VII.1 [34] evaluations. Experimental data is taken from the EXFOR library [35]. Lower panel: same for the ${ }^{237} \mathrm{U}(\mathrm{n}, \gamma)^{238} \mathrm{U}$ cross section.

As an illustration, we show in Fig. 5 the ${ }^{238} \mathrm{U}$ fission path as a function of the quadrupole deformation parameter $\beta_{20}$. The $\mathrm{U}$ fission paths have been used to estimate the ${ }^{237} \mathrm{U}$ neutron-induced fission cross section calculation, as shown in Fig. 6, where the ${ }^{237} \mathrm{U}(\mathrm{n}$,f) cross sections obtained with the HFB-14 fission path and level densities (TALYS-HFB14) [31] and the new HFB-D1M fission paths are compared with the JEFF-3.2 [32,33] and ENDF/B-VII.1 [34] evaluations. Details about the HFB-14 fission path and the calculation of the neutron-induced cross section with the TALYS code can be found in
Refs. [25,31]. The new TALYS-D1M calculation is similar to the TALYS-HFB14 [31] optimization but includes the HFB-D1M fission path for the even-even U isotopes. Both TALYS calculations based on the HFB paths are seen to give rather different estimates of the ${ }^{237} \mathrm{U}(\mathrm{n}, \mathrm{f})$ cross section with respect to the phenomenological approach; such deviations reflect the uncertainties linked to the input models and cannot be accounted for by parameter modifications. The impact of such model uncertainties on experimentally unknown cross sections, like for example ${ }^{237} \mathrm{U}(\mathrm{n}, \gamma){ }^{238} \mathrm{U}$, is even more significant, as shown in Fig. 6 (lower panel).

\section{Conclusion}

Decompressed matter from binary NS mergers remains a viable and robust site for the r-process. This robustness, which is compatible with the unique, solar-like abundance pattern of the elements heavier than Ba observed in metalpoor stars, supports the possible creation of these elements by fission recycling in NS merger ejecta. However, the estimated abundance distribution remains rather sensitive to the adopted nuclear models.

A continued effort to improve our predictions of the reaction and $\beta$-decay rates, including their statistical and systematic uncertainties, for nuclei far away from stability is obviously required. The reliability of our predictions today is still far from being at the level of the requirements in nuclear astrophysics applications. Priority should be given to a better description of the ground-state, fission and $\beta$-decay properties, but also nuclear level densities, optical potential and $\gamma$-ray strength functions. A huge amount of work is still needed to take full advantage of the development of state-of-the-art microscopic models in building global universal models that include as much as possible the microscopic character of quantum physics. This effort to improve the microscopic nuclear predictions is concomitant with new development aiming at improving the description of the reaction mechanisms, including the equilibrium, pre-equilibrium and direct capture processes. This theoretical work requires simultaneously new measurements of structure properties far away from stability, but also reaction cross sections on stable targets and any experiments that can provide new insight on the numerous ingredients of the reaction models and their extrapolation far away from stability.

S.G. is F.N.R.S. research associate.

\section{References}

[1] M. Arnould, K. Takahashi, Rep. Prog. Phys. 62, 395 (1999)

[2] M. Arnould, S. Goriely, K. Takahashi, Phys. Rep. 450, 97 (2007)

[3] H.-T. Janka, Ann. Rev. Nuc. Part. Science 62, 407 (2012)

[4] S. Wanajo, H.-T. Janka, B. Müller, Astrophys. J. Lett. 726, L15 (2011)

[5] B.S. Meyer, Astrophys. J. 343, 254 (1989)

[6] O. Just, A. Bauswein, R. Ardevol Pulpillo, S. Goriely, H.-T. Janka, Mon. Not. Roy. Astron. Soc. 448, 541 (2015) 
[7] A. Bauswein, S. Goriely, H.-T. Janka, Astrophys. J. 773, 78 (2013)

[8] I.U. Roederer, Astrophys. J. 732, L17 (2011)

[9] S. Wanajo, et al., Astrophys. J. Lett. 789, L39 (2014)

[10] M. Dominik, et al., Astrophys. J. 759, 52 (2012)

[11] E. Vangioni, S. Goriely, F. Daigne, P. François, K. Belczynski, Month. Not. Roy. Astro. Soc. 455, 17 (2015)

[12] S. Goriely, N. Chamel, J. M. Pearson, Phys. Rev. 93, 034337 (2016)

[13] G. Audi, et al., Chinese Physics C 36, 1287 (2012)

[14] S. Goriely, S. Hilaire, M. Girod, S. Péru, Phys. Rev. Lett. 102, 242501 (2009)

[15] P. Möller, A.J. Sierk, T. Ichikawa, H. Sagawa, At. Data Nucl. Data Tables 109, 1 (2016)

[16] S. Goriely, R. Capote, Phys. Rev. C 89, 054318 (2014)

[17] D. Neudecker, R. Capote, H. Leeb, Nucl. Instrum. \& Meth. Phys. Res. A 723, 163 (2013)

[18] E. Bauge, P. Dossantos-Uzarralde, J. Korean Phys. Soc. 59, 1218 (2011)

[19] M. Bender, P.-H. Heenen, P.-G. Reinhard, Rev. Mod. Phys. 75, 121 (2003)

[20] M.V. Stoitsov, J. Dobaczewski, W. Nazarewicz, S. Pittel, D.J. Dean, Phys. Rev. C 68, 054312 (2003)

[21] N. Wang, M. Liu, X. Wu, J. Meng, Phys. Lett. B 734, 215 (2014)
[22] S. Goriely, M. Arnould, Astron. Astrophys. 262, 73 (1992)

[23] A. F. Fantina, N. Chamel, J. M. Pearson, S. Goriely, Astron. Astrophys. 559, A128 (2013)

[24] S. Goriely, M. Samyn, J.M. Pearson, Phys. Rev. C 75, 064312 (2007)

[25] S. Goriely, Eur. Phys. J. A 51, 22 (2015)

[26] J.-P. Delaroche, M. Girod, H. Goutte, J. Libert, Nucl. Phys. A 771, 103 (2006)

[27] N. Dubray, D. Regnier, Computer Phys. Comm. 183, 2035 (2012)

[28] R. Rodriguez-Guzman, L. M. Robledo, Phys. Rev. C 89, 054310 (2014)

[29] R. Capote, et al., Nuclear Data Sheets 110, 3107 (2009). See also http://www-nds.iaea. org/RIPL-3

[30] B.-N. Lu, J. Zhao, E.-G. Zhao, S.-G. Zhou, Phys. Rev. C 89, 014323 (2014)

[31] S. Goriely, S. Hilaire, A.J. Koning, R. Capote, Phys. Rev. C 83, 034601 (2011)

[32] M.J. Lopéz Jiménez, B. Morillon, P. Romain, Ann. Nucl. Energy 32, 195 (2005)

[33] A. Santamarina, et. al., "The JEFF-3.1.1 Nuclear Data Library", JEFF report 22, OECD 2009

[34] M.B. Chadwick, et al., Nuclear Data Sheets 112, 2887 (2011)

[35] International Network of Nuclear Reaction Data Centres (NRDC), EXFOR database. Available at http: //www-nds.iaea.org/exfor/exfor.htm 